\title{
Constructive Proof of Localization in the Anderson Tight Binding Model
}

\author{
J. Fröhlich ${ }^{1 \star}$, F. Martinelli ${ }^{2}$, E. Scoppola ${ }^{3}$, and T. Spencer ${ }^{1 \dagger}$ \\ 1 The Institute for Advanced Study, Princeton, NJ 08540, USA \\ 2 Dipartimento di Matematica, Università di Roma "La Sapienza", Piazzale A. Moro, 2, I-00185 Rome, \\ Italy \\ 3 Dipartimento di Fisica, Università di Roma "La Sapienza", Piazzale A. Moro, 2, I-00185 Rome, Italy
}

\begin{abstract}
We prove that, for large disorder or near the band tails, the spectrum of the Anderson tight binding Hamiltonian with diagonal disorder consists exclusively of discrete eigenvalues. The corresponding eigenfunctions are exponentially well localized. These results hold in arbitrary dimension and with probability one. In one dimension, we recover the result that all states are localized for arbitrary energies and arbitrarily small disorder. Our techniques extend to other physical systems which exhibit localization phenomena, such as infinite systems of coupled harmonic oscillators, or random Schrödinger operators in the continuum.
\end{abstract}

\section{Introduction and Outline of Paper}

In this paper we analyze the spectral properties of Anderson's tight binding Hamiltonian, $H$, with diagonal disorder, [1]. This operator describes the dynamics of a quantum mechanical particle moving under the influence of a random potential, $v$. For convenience, we study the discrete case, where the particle may hop on a lattice $\mathbb{Z}^{v}$, but our techniques can be extended to continuous systems. Our main result asserts completeness of the point spectrum and exponential decay of eigenfunctions of $H$ in the band tails, or throughout the spectrum of $H$ provided the disorder is large. This result holds with probability one and in arbitrary dimension $v$. Related results have recently been announced by Ya. Gol'dsheid, but his proofs do not seem to have appeared, yet. In one dimension we may combine our techniques

* Work supported in part by National Science Foundations grant MCS-8108814 (A03). Permanent address: Theoretical Physics, ETH-Hönggerberg, CH-8093 Zürich, Switzerland

$\dagger$ Work supported in part by National Science Foundation grant DMR 81-00417. Permanent address: Courant Institute of Mathematical Sciences, New York University, 251 Mercer Street, New York, NY 10012, USA 
with the positivity of the Lyapunov exponent to recover the result that localization persists at arbitrary energies and for arbitrarily small disorder.

The Hilbert space, $\mathfrak{H}$, of the system studied in later sections is given by

$$
\mathfrak{H}=l_{2}\left(\mathbb{Z}^{v}\right)
$$

and the tight binding Hamiltonian by

$$
H \equiv H(v) \equiv-\Delta+v,
$$

where $\Delta$ is the finite difference Laplacian (with diagonal elements set to zero), i.e. for $i, j$ in $\mathbb{Z}^{v}$,

$$
\Delta_{i j}= \begin{cases}1, & \text { if }|i-j|=1 \\ 0, & \text { otherwise }\end{cases}
$$

and $v$ is the random potential. More precisely

$$
v=\{v(j)\}, \quad j \in \mathbb{Z}^{v},
$$

where the $v(j)$ are independent, identically distributed real random variables, and $v$ acts as a multiplication operator on $\mathfrak{H}$. The distribution of $v(j)$ has a density $g(v(j))$. If $F$ is a function on the probability space, $\Omega$, of all potentials, we denote its average, or expectation, by

$$
\bar{F} \equiv \int_{\Omega} F(v) \prod_{j \in \mathbb{Z}^{v}} g(v(j)) d v(j) \equiv \int_{\Omega} F(v) d P(v)
$$

We let

$$
\delta=\{\sup g(v)\}^{-1}
$$

be a measure of the disorder of our system.

It is well known that the spectrum, $\sigma$, of the Hamiltonian $H$ is given by

$$
\sigma(H)=\sigma(-\Delta)+\sigma(v)=[-2 v, 2 v]+\operatorname{supp} g,
$$

with probability one [2]. Here

$$
A+B \equiv\{a+b: a \in A \subset \mathbb{R}, b \in B \subset \mathbb{R}\} .
$$

The spectrum of $H$ can be decomposed into pure point spectrum, $\sigma_{\mathrm{pp}}(H)$, absolutely continuous spectrum, $\sigma_{\mathrm{ac}}(H)$, and singular continuous spectrum, $\sigma_{\mathrm{sc}}(H)$. Let $T_{E_{0}}$ be the union of the intervals $\left(-\infty, E_{0}\right)$ and $\left(E_{0}, \infty\right)$. Our main result can be rephrased as follows: Suppose that the density $g$ is a bounded function. If $\delta+E_{0}$ is large enough (depending on $v$ ) then

$$
\begin{aligned}
& \sigma_{\mathrm{ac}}(H) \cap T_{E_{0}}=\sigma_{\mathrm{sc}}(H) \cap T_{E_{0}}=\phi, \\
& \text { i.e. } \quad \sigma(H) \cap T_{E_{0}}=\sigma_{\mathrm{pp}}(H) \cap T_{E_{0}},
\end{aligned}
$$

with probability one, and for arbitrary $v$. Furthermore, if supp $g$ is large enough

$$
\sigma_{\mathrm{pp}}(H) \cap T_{E_{0}} \neq \phi,
$$

and eigenfunctions corresponding to eigenvalues in $\sigma_{\mathrm{pp}}(H) \cap T_{E_{0}}$ have exponential decay. 
The strategy of our proof is rooted in several earlier results and methods.

(i) Exponential decay estimates on the Green's function $[H-E-i \varepsilon]_{j l}^{-1}$ of $H$, for any fixed $E \in T_{E_{0}}, \varepsilon \neq 0$. In [3], Fröhlich and Spencer established exponential decay of the Green's function

$$
G(E+i \varepsilon ; j, l)=[H-E-i \varepsilon]_{j l}^{-1}
$$

for any fixed $E \in T_{E_{0}}$, with probability one, provided $\delta+E_{0}$ is sufficiently large. Absence of diffusion was shown to follow from this result. The decay estimates on $G$ are based on an inductive perturbative analysis which shows that "tunnelling at a fixed energy" over long distances is unlikely. A brief description of the techniques of [3] appears in Sect. 2.

While the methods of [3] are strong enough to construct localized states and prove the existence of dense point spectrum in $T_{E_{0}}$, for large $\delta+E_{0}$, (these results are described in [4]) they are not sufficient to prove completeness of point spectrum in $T_{E_{0}}$. In order to prove a result like (1.7) one would like to show that tunnelling over long distances is very unlikely, for all energies $E \in \sigma(H) \cap T_{E_{0}}$ simultaneously. This will be shown in Sects. 3 and 4, extending ideas in $[3,4]$.

(ii) Absence of absolutely continuous spectrum in $T_{E_{0}}$. Recently, Martinelli and Scoppola [5] proved that the exponential decay of the Green's function implies the absence of absolutely continuous spectrum. Their proof combines the exponential decay estimates for $G(E+i \varepsilon ; j, l)$ with the fact that generalized eigenfunctions of $H$ are polynomially bounded [6]. This fact plays an important role in the present analysis as well.

(iii) Completeness of point spectrum in a certain range of energies for a Hamiltonian with hierarchical random potential. In [7] Jona-Lasinio, Martinelli and Scoppola proved that for a Hamiltonian with hierarchical random potential the spectrum is pure point near the lower band edge. Such models are already remarkable at the deterministic level, as they can essentially be solved exactly. The potential has minima separated by barriers of rapidly increasing width in such a way that the potential is approximately self-similar over a sequence of length scales. In the stochastic version of these models the heights of the minima fluctuate randomly. The tunnelling processes in the hierarchical models mimick tunnelling processes in Anderson's model studied in this paper. The strategy of our proof of localization, as described in Sect. 3, is patterned closely after the work in [7].

In Sect. 4 the key probabilistic lemma is proven. The proof uses the methods of [3].

We now make the above remarks more precise.

Definition 1. We say that a function $\psi$ on $\mathbb{Z}^{v}$ is a generalized eigenfunction of the Hamiltonian $H=H(v)$, defined in (1.2), corresponding to a generalized eigenvalue $E(v)$ iff $\psi$ is a polynomially bounded solution to the equation

$$
H(v) \psi=E(v) \psi
$$

Definition 2. Given a self-adjoint operator $H$ on the Hilbert space $\mathfrak{H}$, let $E_{H}(\cdot)$ denote 
the spectral projection for $H$. We define a Borel measure

$$
\rho(B)=\sum_{n=0}^{\infty} 2^{-n}\left\langle e_{n}, E_{H}(B) e_{n}\right\rangle,
$$

where $\langle\cdot, \cdot\rangle$ denotes the scalar product on $\mathfrak{H},\left\{e_{n}\right\}_{n=0}^{\infty}$ is an orthonormal basis of $\mathfrak{H}$, and $B$ is an arbitrary measurable subset of $\mathbb{R}$. Every Borel measure equivalent to $\rho$ is called a spectral measure.

In our proof of localization we use the following result. (For proofs see [6].)

Theorem 1.1. For almost every potential $v$, there exists a spectral measure $\rho_{v}$ such that almost every energy, $E$, with respect to $\rho_{v}$, is a generalized eigenvalue corresponding to a generalized eigenfunction (in the sense of Definition 1).

Theorem 1.2. If I is an interval with the property that every generalized eigenfunction corresponding to a generalized eigenvalue in I decays exponentially fast, then

$$
\sigma_{a c}(H) \cap I=\sigma_{s c}(H) \cap I=\phi,
$$

i.e. the spectrum of $H$ in $I$ is pure point.

The proof of Theorem 1.2 is obvious.

Theorem 1.3. Let $H=H(v)$ be the tight binding Hamiltonian defined in (1.2). If $E_{0}+\delta$ is sufficiently large then every generalized eigenfunction of $H(v)$ corresponding to a generalized eigenvalue $E(v)$, with $|E(v)|>E_{0}$, decays exponentially fast.

Remark. As shown in [5], the set of generalized eigenvalues in $T_{E_{0}}$ depends nontrivially on the potential configuration $v$. In particular, any fixed energy $E \in T_{E_{0}}$ is not a generalized eigenvalue with probability one.

Combining Theorems 1.1 through 1.3 we obtain our

Main Result. For $E_{0}+\delta$ large enough, the spectrum of $H(v)$ outside $\left[-E_{0}, E_{0}\right]$ is pure point, and the eigenfunctions of $H(v)$ corresponding to eigenvalues outside $\left[-E_{0}, E_{0}\right]$ decay exponentially.

As a corollary of our main result we get some information about the spreading of wave packets under the dynamics $e^{i t H(v)}$, as $t \rightarrow \pm \infty$. Let

$$
r^{2}(t, \psi) \equiv \sum_{j \in \mathbb{Z}^{v}}|j|^{2}\left|\left(e^{i t H(v)} \psi\right)(j)\right|^{2}
$$

Corollary. If $E_{0}+\delta$ is sufficiently large and if $\psi$ is a wave function with the property that $|\psi(j)|$ is of rapid decrease in $|j|$, and

$$
E_{H(v)}\left(\left[-E_{0}, E_{0}\right]\right) \psi=0
$$

then

$$
r^{2}(t, \psi) \leqq \text { const }
$$

uniformly in $t$, with probability one.

The proof of this Corollary follows directly from Theorem 5.2 of [7] and our Main Result and is therefore omitted. (For an earlier, related but weaker result see [3].)

Our results are reviewed in [8]* 
Next, we show how Theorem 1.3 (exponential decay of generalized eigenfunctions) can be reduced to proving exponential decay of Green's functions at energies corresponding to a generalized eigenvalue in $T_{E_{0}}$. We define

$$
G_{\Lambda}(z ; i, j) \equiv\left[H_{\Lambda}-z\right]_{i j}^{-1}=\left\langle\delta_{i},\left[H_{\Lambda}-z\right]^{-1} \delta_{j}\right\rangle,
$$

where $z$ is a complex number, $\left(H_{\Lambda}\right)_{i j}=H_{i j}$ if $i$ and $j$ belong to a subset $\Lambda$ of $\mathbb{Z}^{v}$, and $\left(H_{\Lambda}\right)_{i j}=0$, otherwise. Moreover $\delta_{i}(l)$ is the Kronecker $\delta$-function. Let

$$
\Gamma=\partial \Lambda
$$

be the set of nearest-neighbor pairs $\langle i, j\rangle$ with exactly one site in $\Lambda$. We identify $\Gamma$ with an operator whose matrix elements are

$$
\Gamma_{i j}= \begin{cases}1, & \langle i, j\rangle \in \Gamma \\ 0, & \text { otherwise }\end{cases}
$$

Clearly

$$
\mathfrak{H}=l_{2}(\Lambda) \oplus l_{2}\left(\Lambda^{c}\right)
$$

and

$$
H=H_{\Lambda} \oplus H_{\Lambda^{c}}-\Gamma .
$$

Let $E(v)$ be a generalized eigenvalue and $\psi$ the corresponding generalized eigenfunction of $H(v)$. Then, for any subset $\Lambda$ of $\mathbb{Z}^{v}$, the time-independent Schrödinger equation for $\psi$ can be written in the form

$$
([H+\Gamma-E(v)] \psi)(j)=(\Gamma \psi)(j),
$$

and hence, using (1.14) and estimates on $G_{\Lambda}(E(v) ; j, l)$.

$$
\psi(j)=\sum_{l, l^{\prime} \in \partial \lambda} G_{\Lambda}(E(v) ; j, l) \Gamma_{l l^{\prime}} \psi\left(l^{\prime}\right)
$$

for $j \in \Lambda$.

In order to prove exponential decay of $\psi$ outside some finite box, it is convenient to apply (1.15) to a sequence of annular regions

$$
A_{k}=\Lambda_{k+1} \backslash \tilde{\Lambda}_{k},
$$

where $\Lambda_{k}$ and $\tilde{X}_{k}$ are cubes centered at the origin of $\mathbb{Z}^{v}$ with sides parallel to the lattice axes of length $8 d_{k}, 4 d_{k}$, respectively, and

$$
d_{k}=\exp \left[\beta(5 / 4)^{k}\right], \quad k=0,1,2, \ldots,
$$

for some constant $\beta$ chosen later. Exponential decay of $\psi$ then follows from exponential decay of $G_{A_{k}}(E(v) ; j, l)$, for all sufficiently large $k$.

In Sect. 3 we prove that, for large $\delta+E_{0}$, there is a finite integer $k$ such that, for all $k>k$ and all $j$ and $l$ in $A_{k}$, with $|j-l| \geqq d_{k-1} / 5$,

$$
\left|G_{A_{k}}(E(v) ; j, l)\right| \leqq \exp [-m|j-l|], \quad m>0,
$$

with probability one, where $E(v)$ is an arbitrary generalized eigenvalue with $|E(v)|>E_{0} ; m$ depends on $E(v)$. Let $j$ be an arbitrary site. We choose an integer $k$ 
such that $j \in A_{k}=\Lambda_{k+1} \backslash X_{k}$ and

$$
\operatorname{dist}\left(j, \Gamma_{k}\right) \geqq \frac{1}{3}|j| \geqq \frac{1}{5} d_{k-1},
$$

where

$$
\Gamma_{k}=\partial A_{k} .
$$

A simple geometrical consideration shows that an integer $k$ with these properties can always be chosen. One then applies Eq. (1.15) with $\Lambda=A_{k}$ and $\Gamma=\Gamma_{k}$. Exponential decay of $\psi$ then follows from (1.17) and (1.18) and the fact that $\psi$ is polynomially bounded.

Straightforward modifications of the arguments just given show that the decay rate of $|\psi(j)|$, as $|j| \rightarrow \infty$, is bounded from below by the decay rate $m=m(E(v))$ of the Green's function $G_{A_{k}}(E(v) ; j, l)$, for $k>\bar{k}$.

Remark. The proof of the basic estimate (1.17) consists of two parts [3]: In a deterministic part, one characterizes the potential configurations, $v$, which are "nonsingular on scale $k$," in the sense that $G_{A_{k}}(E(v) ; j, l)$ decays exponentially in $|j-l|$, for $|j-l|>0\left(d_{k}\right)$. In a probabilistic part one shows that, with probability one, potential configurations are actually non-singular on scale $k$, for $k$ large enough.

One possible characterization of typical configurations is the following (see Sect. 6): Let $E(v)$ be a generalized eigenvalue, with $|E(v)|>E_{0}$ and $E_{0}+\delta$ large enough. Then (with probability one)

(a) $\operatorname{dist}\left(\sigma\left(H_{\Lambda_{k}}(v)\right), E(v)\right) \leqq e^{-\left(d_{k}^{\gamma} / 3\right)}$, for $k$ large enough;

(b) $\operatorname{dist}\left(\sigma\left(H_{A_{k}}(v)\right), E(v)\right)>e^{-\left(d_{k}^{\gamma} / 3\right)}$, for $k$ large enough. Here $0<\gamma<1$.

(c) (1.17) holds.

This characterization of the typical configurations is analogous to the one given in [7] for the hierarchical random potentials. We shall, however, use a somewhat different characterization of typical configurations which is technically more convenient.

Our paper is organized as follows.

In Sect. 2 we review results on the density of states, due to Wegner [9], and the decay of the Green's functions, $G_{A_{k}}$, proven in [3]. We also describe an extension of the inductive construction of [3], which is useful for the analysis of the onedimensional tight binding Hamiltonian. These results are needed in Sects. 3-5.

In Sect. 3 we reduce the proof of our basic decay estimate (1.17) to several technical lemmas, in particular some kind of non-resonance condition for the spectra of the Hamiltonians $H_{c}$ and $H_{c^{\prime}}$, where $c$ ranges over some family of subsets of $\tilde{\Lambda}_{k}$ (cubes of different sizes), and $c^{\prime}$ ranges over a family of subsets of the annulus $A_{k}$, for all sufficiently large $k$.

In Sect. 4 we prove the main technical lemma used in Sect. 3. This requires some fairly lengthy probabilistic estimates.

In Sect. 5, we show how one may recover the results on localization in one dimension [10-12] by making use of the positivity of Liapunov exponents in order to prove the decay estimates on Green's functions required in our approach to localization theory.

In Sect. 6, we briefly describe some other physical systems which exhibit localization and which could be analyzed with the help of our techniques. 
Remark. After completion of our paper we were informed of two new proofs of localization, based directly on the decay estimates of reference 3 and combining them with general functional analysis - in addition to the announced proof of Ya. Gol'dsheid. One proof is due to Simon and Wolff. We thank B. Simon for informing us of their work and sending us a preprint prior to publication. The second proof is due to Delyon, Lévy and Souillard.

Our own proof, though technically more complicated, may have the advantage of having a very simple and transparent strategy, of being more constructive and extending to other problems, like random Schrödinger operators in the continuum; see [13] for some results on this case.

\section{Review of Previous Methods and Results}

In this section we describe configurations of non-singular potentials, $v$, for which we prove exponential decay of the Green's function, for fixed E. The regularity properties of these potentials, for a fixed value of $E$, are expressed inductively, the induction extending over a sequence of rapidly increasing length scales, $d_{k},[3]$. We also recall Wegner's result on the density of states of tight binding Hamiltonians [9] which is important for the probability estimates in Sects. 3 and 4. Finally we sketch a "renormalized" inductive construction of non-singular configurations designed to prove decay of the Green's function for a larger range of energies, in particular for all energies when $v=1$.

Given an energy $E$ and a subset $\Lambda$ of $\mathbb{Z}^{v}$, we define a decreasing family of singular sets $S_{k}(E, v, \Lambda)$, with the property that

$$
S_{0} \supseteq S_{1} \supseteq S_{2} \supseteq \cdots .
$$

The set $S_{n}(E, v, \Lambda)$ is the set of sites where the potential $v$ is "singular of strength $\geqq n$ " and is defined inductively. We set

$$
S_{0}(E, v, \Lambda)=\left\{j \in \Lambda:|v(j)-E| \leqq 2 v+m_{0}\right\},
$$

with

$$
0<m_{0}=m_{0}(E) \sim \ln |E| .
$$

Notice that if $\delta$ or $|E|$ is large, $S_{0}(E, v, \Lambda)$ is a subset of $\Lambda$ of very small density. If $S_{0}(E, v, \Lambda)=\phi$, then we can prove exponential decay of the Green's function $G_{\Lambda}(E ; j, l)$ in $|j-l|$ by perturbation theory: The Laplacian $\Delta$ can be regarded as a small perturbation of $v-E-i \varepsilon$. It is easy to see that

$$
\begin{aligned}
& \left|G_{\Lambda}(E+i \varepsilon ; j, l)\right|=\left|\sum_{\substack{\omega: j \rightarrow l \\
\omega \subset \Lambda}} \prod_{i \in \Lambda}(v(i)-E-i \varepsilon)^{-n_{\mathbf{\imath}}(\omega)}\right| \\
& \leqq \sum_{\omega: j \rightarrow l} \prod_{i \in \mathbb{Z}^{v}}\left(2 v+m_{0}\right)^{-n_{i}(\omega)} \\
& =\left(-\Delta+2 v+m_{0}\right)_{j l}^{-1},
\end{aligned}
$$

which decays exponentially fast in $|j-l|$. Here $\omega$ denotes a nearest-neighbor walk starting at $j$ and ending at $l$, and $n_{j}(\omega)$ is the total number of visits of $\omega$ at $j$; see 
$[14,3]$. (The walks $\omega$ just serve to label terms in the Neumann series expansion of $[-\Delta+v-E-i \varepsilon]_{j l}^{-1}$ in $\Delta$.)

It is necessary to extend the decay estimate (2.2) to Green's functions, $G_{\Lambda}$, with the property that $\Lambda \cap S_{0} \neq \phi$. For this purpose we define the sets $S_{k}=S_{k}(E, v, \Lambda) \subset \Lambda$ inductively as follows.

$$
S_{k+1}=S_{k} \backslash \bigcup_{\alpha} C_{k}^{\alpha}
$$

where $\bigcup_{\alpha} C_{k}^{\alpha}$ is the maximal union of components $C_{k}^{\alpha} \subseteq S_{k}$ which are regular at the $k^{\text {th }}$ scale; more precisely $\left\{C_{k}^{\alpha}\right\}_{\alpha=1,2,3, \ldots}$ is the maximal family of disjoint components satisfying

\section{Condition $k$.}

(a) $C_{k}^{\alpha} \subseteq \Lambda$, and $\operatorname{diam}\left(C_{k}^{\alpha}\right) \leqq d_{k}$,

(b) $\operatorname{dist}\left(C_{k}^{\alpha}, S_{k} \backslash C_{k}^{\alpha}\right) \geqq 2 d_{k}^{5 / 4}=2 d_{k+1}$

(c) $\operatorname{dist}\left(\sigma\left(H_{\bar{C}_{k}^{\alpha}}\right), E\right) \geqq e^{-\sqrt{d_{k}}}$.

Here "diam" denotes the diameter of a set,

$$
\operatorname{dist}(A, B)=\inf _{\substack{a \in A \\ b \in B}}|a-b|,
$$

and

$$
d_{k}=\exp \left[\beta\left(\frac{5}{4}\right)^{k}\right], \quad k=0,1,2, \ldots
$$

Moreover, $\bar{C}_{k}^{\alpha} \subseteq \Lambda$ is a lattice set containing $C_{k}^{\alpha}$ with the property that

$$
\operatorname{dist}\left(C_{k}^{\alpha}, \partial \bar{C}_{k}^{\alpha} \backslash \partial \Lambda\right) \geqq 4 d_{k}
$$

and is further specified as follows. Let $\mathfrak{C}_{m}$ be the collection of lattice cubes with sides parallel to the lattice axes and of length $2^{m}$ which are centered at the sites of $2^{m-1} \mathbb{Z}^{v}$. We require that

$$
\bar{C}_{k}^{\alpha}=c \cap \Lambda,
$$

where $c$ belongs to $\mathfrak{C}_{n(k)}$ and $n(k)$ is determined by

$$
2^{n(k)} \geqq 10 d_{k} \geqq 2^{n(k)-1} \text {. }
$$

Remarks. In [3] only condition (2.8), but not condition (2.9), was imposed on the choice of $\bar{C}_{k}^{\alpha}$. It is easily verified that condition (2.9) can be imposed without invalidating any of the arguments in [3]. In the following condition (2.9) will turn out to be convenient.

We also note that we could have defined the numbers $d_{k}$ to be powers of 2, i.e. $d_{k}=2^{\delta_{k}}$, for some integers $\delta_{k}$. In this case the lattice sets $\Lambda, \Lambda_{k}, X_{k}$ are all centered at 0 and have sides of length $2^{n}, n=1,2,3, \ldots$, parallel to the lattice axes. We can then choose the cube $c$ in (2.9) to lie entirely inside $\Lambda$, and hence every $\bar{C}_{k}^{\alpha} \subset \Lambda$ belongs to some $\mathfrak{C}_{n(k)}$. 
Definition 3. Given $\Lambda$, a set $A \subset \Lambda$ is said to be $k$-admissible iff

$$
\partial A \cap \bar{C}_{l}^{\alpha} \subset \partial \Lambda, \quad l=0,1, \ldots, k .
$$

Remark. If $S_{k}(E, v, \Lambda)$ is empty and $A$ is a $(k-1)$-admissible subset of $\Lambda$, then $S_{k}(E, v, A)$ is empty, as well.

It is easy to see that $k$-admissibility of lattice regions is a very mild requirement.

Lemma [3]. If $D_{1}$ and $D_{2}$ are two subsets of $\Lambda$ such that $D_{1} \supset D_{2}$ and

$$
\operatorname{dist}\left(D_{2}, D_{1}^{c} \cap \Lambda\right) \geqq 30 d_{k},
$$

then there is a $k$-admissible region $A$ with

$$
D_{1} \supset A \supset D_{2} .
$$

The idea behind the proof is to deform the boundary of $D_{2}$ so as to avoid singular components $\bar{C}_{i}^{\alpha}, i \leqq k$, in such a way that the resulting set remains within $D_{1}$ but contains $D_{2}$. Since diam $\left(\bar{C}_{i}^{\alpha}\right) \leqq 20 d_{i}<30 d_{k}$, for all $i \leqq k$, and by $(2.10)$, this can be achieved. For details of the proof, see Appendix $D$ of [3].

Next, we recall the basic decay estimates for the Green's function of the tight binding Hamiltonian.

Theorem 2.1. [3]. If the constant $\beta$ in the Definition (2.7) of $\left\{d_{k}\right\}$ is chosen sufficiently large, then there is a positive constant $m \geqq \frac{1}{2} m_{0}$ such that if $S_{k}(E, v, \Lambda)=\phi$, then

$$
\left|G_{\Lambda}(E+i \varepsilon ; j, l)\right| \leqq e^{-m|J-l|},
$$

for all $j$ and $l$ in $\Lambda$ satisfying $|j-l| \geqq \frac{1}{5} d_{k}$.

This result is proven by induction in $k$. Singular components, $C_{i}^{\alpha}$, are inductively incorporated into the system in $\Lambda$ by means of simple perturbation theory.

The next result, due to Wegner, is used in our probability estimates.

Theorem 2.2 [9]. For any finite region $\Lambda \subset \mathbb{Z}^{v}$ and any $\kappa>0$,

$$
\operatorname{Prob}\left\{v: \operatorname{dist}\left(\sigma\left(H_{\Lambda}(v)\right), E\right) \leqq \kappa\right\} \leqq \varepsilon(\kappa, E, \delta)|\Lambda|,
$$

where

$$
\varepsilon(\kappa, E, \delta) \equiv \min \left(2 \kappa \delta^{-1}, \sqrt{2 \kappa} \bar{\rho}(E, \delta)\right)
$$

with

$$
\delta^{-1}=\sup g(\cdot) ; \quad \bar{\rho}(E, \delta) \rightarrow 0, \quad \text { as } \quad \delta+|E| \rightarrow \infty .
$$

Moreover, $|\Lambda|$ denotes the number of sites in $\Lambda$. [8].)

(See Lemma 2.4 and Appendix $C$ of [3] for this variant of Theorem 2.2; see also

Corollary 2.3. If $\Lambda$ and $\Lambda^{\prime}$ are two disjoint lattice regions, then

$$
\operatorname{Prob}\left\{v: \operatorname{dist}\left[\sigma\left(H_{\Lambda}(v)\right), \sigma\left(H_{\Lambda^{\prime}}(v)\right)\right] \leqq \kappa\right\} \leqq 2 \kappa \delta^{-1}|\Lambda|\left|\Lambda^{\prime}\right|
$$

The proof of Corollary 2.3 follows easily from Theorem 2.2 by noticing the facts that the eigenvalues of $H_{\Lambda}$ are statistically independent from the eigenvalues of $H_{\Lambda^{\prime}}$ 
and that

$$
\text { \# }\left\{\text { eigenvalues of } H_{\Lambda^{\prime}}\right\}=\operatorname{dim} l_{2}\left(\Lambda^{\prime}\right)=\left|\Lambda^{\prime}\right| \text {. }
$$

We conclude Sect. 2 with an outline of a coarse-grained, or renormalized version of the inductive scheme of [3] which we use to prove localization in one dimension for arbitrary energies and arbitrarily small disorder. We note, however, that our renormalized inductive scheme should also be useful in higher dimensions as a means to understand localization for energies close to a mobility edge. For another, simple example of a coarse-grained version of the scheme in [3] see also [13].

The key idea on which our renormalized inductive scheme is based is to replace singular sites by singular blocks of sites and to trade the decay estimate (2.2) for $G_{\Lambda}(E ; j, l)$ on regions $\Lambda$ for which $S_{0}(E, v, \Lambda)=\phi$ for an estimate on the decay of the Green's function associated with large blocks of sites which one attempts to prove e.g. by nonperturbative methods. One then expands in couplings between different blocks. The expected effect of this renormalization procedure is to drive the system towards a "large-disorder fixed point."

We now describe the basic hypothesis of our improved method in more detail. Suppose that, for a sufficiently large block $B$, a cube centered at 0 with sites of length $2 l$ parallel to the lattice axes, and all energies, $E$, in an interval $I$

$$
\begin{gathered}
\operatorname{Prob}\left\{v: \sum_{y \in \partial B}\left|G_{B}(E ; x, y)\right|<a, \text { for }|x-y| \geqq \frac{1}{4} l, y \in \partial B\right\} \\
\geqq 1-\varepsilon(v, l, I, a)
\end{gathered}
$$

for some $a<1$ which may depend on $I$ and $\delta$, with $\varepsilon(v, l, I, a) \rightarrow 0$, as $l \rightarrow \infty$ and $|I| \rightarrow 0$.

We now cover $\mathbb{Z}^{v}$ with translates of the cube $B$ centered at the sites of the lattice $l \mathbb{Z}^{v}$. The cube centered at the site $j \in l \mathbb{Z}^{v}$ is denoted by $B_{j}$. We define

and

$$
\begin{aligned}
& S_{0}^{R}(E, v, \Lambda) \equiv\left\{j \in l \mathbb{Z}^{v}: \sum_{y \in \partial B_{J}}\left|G_{B_{j}}(E ; x, y)\right| \geqq a\right. \\
&\left.\quad \text { for some } x \in B_{j} \text { with }|x-y| \geqq \frac{1}{4} l, y \in \partial B_{j}\right\}
\end{aligned}
$$

By iterating the identity

$$
\bar{S}_{0}^{R}(E, v, \Lambda)=\bigcup_{j \in S_{0}^{R}(E, v, \Lambda)} B_{j} .
$$

$$
G_{\Lambda}(z ; x, y)=G_{B_{J}}(z ; x, y)+\sum_{\left\langle u, u^{\prime}\right\rangle \in \partial B_{j}} G_{B_{J}}(z ; x, u) G_{\Lambda}\left(z ; u^{\prime}, y\right)
$$

with $B_{j}$ chosen such that $x \in B_{j}$ and $\operatorname{dist}\left(x, \partial B_{j}\right) \geqq \frac{l}{4}$, we may easily show that if $S_{0}^{R}(E, v, \Lambda)=\phi$, then $\left|G_{\Lambda}(E+i \varepsilon ; x, y)\right|$ decays exponentially with decay rate $\propto l^{-1} \ln a^{-1}$, for all $\varepsilon \neq 0$; (see Sect. 3 of [3] for a very similar argument). This estimate replaces (2.2).

Sets of blocks, $S_{k}^{R}$ and $\bar{S}_{k}^{R}$, which are singular on the $k^{\text {th }}$ scale can now be defined in a way very similar to the definition of $S_{k}, k=1,2,3, \ldots$ The analogue of Theorem 
2.1 can be proven by the methods of [3]. All arguments in [3] clearly extend to the present situation with only minor changes in notation. This will permit us to establish localization under the assumption that condition (2.14) holds.

The methods just sketched are particularly transparent in one dimension. In this case the blocks $B_{j}$ are intervals of length $2 l$ centered at the sites of $l \mathbb{Z}$. In Sect. 5 we shall derive condition (2.14) from the positivity of the Liapunov exponent. This will prove localization for the one-dimensional tight binding Hamiltonian at arbitrary energies and for arbitrarily weak disorder.

Some combinatorial techniques and estimates from $[15,3]$ will be reviewed in Sect. 4 , where they are needed to prove various probability estimates.

\section{Proof of Localization, Modulo a Technical Lemma}

In this section we complete the proof of Theorem 1.3 (exponential decay of generalized eigenfunctions corresponding to eigenvalues $E(v)$, with $|E(v)| \geqq E_{0}$, for $E_{0}+\delta$ large enough) by proving the exponential decay estimate (1.17) on the Green's function $G_{A_{k}}(E(v) ; i, j)$. Here

$$
A_{k}=\Lambda_{k+1} \backslash \tilde{\Lambda}_{k}
$$

where $\Lambda_{k}$ and $\tilde{\Lambda}_{k}$ are cubes centered at the origin with sides of length $8 d_{k}$ and $4 d_{k}$, respectively, parallel to the lattice axes.

The Main Result stated in Sect. 1 (localization, i.e. completeness of point spectrum, for energies outside $\left[-E_{0}, E_{0}\right]$, for sufficiently large $E_{0}+\delta$ ) follows from Theorem 1.3, as explained in Sect. 1. The "disorder parameter" $\delta$ has been defined in (1.6). The main result of this section is the following theorem.

Theorem 3.1. If $\delta+E_{0}$ is sufficiently large there is a set $\Omega^{\prime}$ of potentials, $v$, of measure one such that, for all $v \in \Omega^{\prime}$ and every generalized eigenvalue $E(v)$, with $|E(v)|>E_{0}$, there exists an integer $\bar{k}=\bar{k}(E(v), v)<\infty$ such that the events

(i) $S_{k-1}\left(E(v), v, \Pi_{k}\right) \neq \phi$ and

(ii) $S_{k-1}\left(E(v), v, A_{k}\right)=\phi, \quad A_{k}=\Lambda_{k+1} \backslash X_{k}$, hold for all $k>k$.

Theorem 3.1 establishes the hypotheses of Theorem 2.1 for $\Lambda=A_{k}$, i.e.

$$
\left|G_{A_{k}}(E(v)+i \varepsilon ; j, l)\right| \leqq e^{-m|j-l|}, \quad m \equiv m(E(v))>0,
$$

for all $j$ and $l$ in $A_{k}$ with $|j-l| \geqq \frac{1}{5} d_{k-1}$. This is precisely the required decay estimate (1.17).

The proof of Theorem 3.1 is based on the following two lemmas.

Lemma 3.2. For every potential $v$, and given $v$, for every generalized eigenvalue $E(v)$, there is a finite integer $\hat{k}=\hat{k}(v, E(v))$ such that, for all $k \geqq \hat{k}$,

$$
S_{k-1}\left(E(v), v, \tilde{\Lambda}_{k}\right) \neq \phi
$$

Remark. The proof of this lemma is almost identical to the proof of Lemma 5.2 of [7] and is included here for completeness.

Proof. Suppose there is a sequence $\left\{k_{i}\right\}_{i=1}^{\infty}$ diverging to $+\infty$ such that $S_{k_{i}-1}\left(E(v), v, X_{k_{i}}\right)=\phi$, for all $i$. Then, by Theorem 2.1,

$$
G_{{X_{k_{i}}}}(E(v) ; j, l) \leqq e^{-m|j-l|},
$$


for some $m=m(E(v))>0$ and all $j$ and $l$ in $\tilde{\Lambda}_{k_{1}}$, with $|j-l| \geqq \frac{1}{5} d_{k_{i}-1}$. The generalized eigenfunction $\psi$ corresponding to $E(v)$ is determined inside $\tilde{X}_{k_{\mathrm{r}}}$ by its value on $\tilde{\Gamma}_{k_{i}} \equiv \partial \tilde{\Lambda}_{k_{i}}$ via the formula

$$
\psi(x)=\sum_{\left\langle y, y^{\prime}\right\rangle \in \tilde{\Gamma}_{k_{\iota}}} G_{\tilde{\lambda}_{k_{l}}}(E(v) ; x, y)\left(\tilde{\Gamma}_{k_{\imath}}\right)_{y y^{\prime}} \psi\left(y^{\prime}\right),
$$

see (1.15). Since $\psi$ is polynomially bounded and by (3.2), we obtain from (3.3) the bound

$$
|\psi(x)| \leqq \text { const } \sum_{y \in \partial X_{k_{\iota}}} e^{-m|x-y|}|y|^{p},
$$

for some finite $p$ and all $x$, with $|x|<d_{k_{i}}$. The right-hand-side of (3.4) tends to 0 , as $i \rightarrow \infty$, for every fixed $x$. Hence $\psi(x)=0$, which contradicts our assumption that $E(v)$ is a generalized eigenvalue. This completes the proof of Lemma 3.2.

To formulate our next lemma we need some more definitions. Let $\mathbb{C}=\bigcup_{m=0}^{\infty} \mathfrak{C}_{m}$ denote the family of lattice cubes on all scales introduced after (2.8).

Definition 4. For a given set $\Lambda \subset \mathbb{Z}^{v}$ and a finite interval $I$, we define

$$
\begin{aligned}
& \mathfrak{B}_{k}(\Lambda, I) \equiv\left\{v: S_{k-1}(E, v, \Lambda) \neq \phi, \text { and for all } c \in \mathfrak{C}\right. \\
&\left.\operatorname{dist}\left(\sigma\left(H_{c \cap \Lambda}\right), E\right) \geqq \exp \left[-d_{k-1}^{\gamma}\right], \text { for some } E \in I\right\} .
\end{aligned}
$$

Here $\gamma$ is some constant, with $0<\gamma<1$.

Remark. In (3.5) one may replace $\mathfrak{C}$ by $\bigcup_{m=0}^{n(\Lambda)} \mathfrak{C}_{m}$, where $n(\Lambda)$ is the smallest integer such that $\Lambda$ is entirely contained in some cube of $\mathfrak{C}_{n(\Lambda)}$.

Lemma 3.3. For sufficiently small $\gamma$ and arbitrary $p<\infty$, there are constants $\delta_{0}(p)$ and $E_{0}=E_{0}(p, \delta)$ such that

$$
\operatorname{Prob}\left(\mathfrak{B}_{k}(\Lambda, I)\right) \leqq|\Lambda| d_{k}^{-p},
$$

provided $\delta>\delta_{0}(p)$ and $|I|$ is sufficiently small, or $I \cap\left[-E_{0}, E_{0}\right]=\phi$, with $E_{0}=$ $E_{0}(p, \delta)$.

Remark. Note that the probability estimate of Lemma 3.3 involves uncountably many energies, namely all $E \in I$, while in the probability estimates of [3] the energy was fixed. The proof of Lemma 3.3 thus requires some work which we present in Sect. 4.

Proof of Theorem 3.1. Since, for $p$ large enough $(p>v)$, the series

$$
\sum_{k=0}^{\infty} \operatorname{Prob}\left(\mathfrak{B}_{k}\left(\widetilde{\Lambda}_{k}, I\right)\right)
$$

converges, if $\delta>\delta_{0}(p)$, or $I \cap\left[-E_{0}, E_{0}\right]=\phi$, with $E_{0}=E_{0}(p, \delta)$, the Borel-Cantelli lemma assures us that with probability one there exists some $\widetilde{k}=\widetilde{k}(v)<\infty$ such that, for $k>\tilde{k}$, the event

$$
S_{k-1}\left(E, v, \Pi_{k}\right) \neq \phi
$$


implies that

$$
\operatorname{dist}\left(\sigma\left(H_{c \cap X_{k}}\right), E\right)<\exp \left[-d_{k-1}^{\gamma}\right]
$$

for some $c \in \mathfrak{C}$.

Exactly the same statement holds, with $A_{k}=\Lambda_{k+1} \backslash \tilde{\Lambda}_{k}$ replacing $\tilde{\Lambda}_{k}$.

Definition 5. We define events $\mathscr{E}_{k}$ by

$$
\begin{aligned}
\mathscr{E}_{k} \equiv\left\{v: \operatorname{dist}\left[\sigma\left(H_{c \cap X_{k}}\right) \cap I, \sigma\left(H_{c^{\prime} \cap A_{k}}\right)\right] \leqq 2 \exp \left[-d_{k-1}^{\gamma}\right],\right. \\
\left.\quad \text { for some } c \text { and } c^{\prime} \text { in } \mathfrak{C}\right\} .
\end{aligned}
$$

By Corollary 2.3,

$$
\operatorname{Prob}\left(\mathscr{E}_{k}\right) \leqq \text { const } \sum_{c, c^{\prime}}|c|\left|c^{\prime}\right| \exp \left[-d_{k-1}^{\gamma}\right],
$$

where the sum on the right-hand-side of (3.8) ranges over cubes $c \in \mathfrak{C}_{m}$ and $c^{\prime} \in \mathfrak{C}_{m^{\prime}}$ intersecting $\tilde{\Lambda}_{k}$ and $A_{k}$, respectively, with $m=0, \ldots, n\left(\tilde{\Lambda}_{k}\right)$ and $m^{\prime}=0, \ldots, n\left(\Lambda_{k+1}\right)$, where $n\left(\tilde{\Lambda}_{k}\right)$ and $n\left(\Lambda_{k+1}\right)$ are as in the remark following Definition 4, above, whence

$$
m, m^{\prime} \leqq 2 \log _{2}\left(\operatorname{diam} \Lambda_{k+1}\right) .
$$

Since the number of cubes $c \in \mathfrak{C}_{m}$ which intersect a fixed box $\Lambda$ is bounded by $2^{-(m-2) v}|\Lambda|$ and by (3.9), it follows that

$$
\sum_{k=0}^{\infty} \operatorname{Prob}\left(\tilde{E}_{k}\right) \text { converges, }
$$

for any $\gamma>0$. By applying the Borel-Cantelli lemma once more we conclude that, with probability one, there exists a constant $k^{\prime}=k^{\prime}(v)$ such that for all $k>k^{\prime}$,

$$
\operatorname{dist}\left[\sigma\left(H_{c \cap \tilde{X}_{k}}\right) \cap I, \sigma\left(H_{c^{\prime} \cap A_{k}}\right)\right] \geqq 2 \exp \left[-d_{k-1}^{\gamma}\right],
$$

for all $c$ and $c^{\prime}$ in $\mathfrak{C}$.

By combining (3.6) and (3.10) we conclude that, for $k>\max \left(\widetilde{k}, k^{\prime}\right)$ and all $E \in I$,

$$
S_{k-1}\left(E, v, \tilde{\Lambda}_{k}\right) \neq \phi \Rightarrow S_{k-1}\left(E, v, A_{k}\right)=\phi .
$$

By Lemma 3.2, we know that if $\delta+E_{0}$ is large enough and $E(v)$ is a generalized eigenvalue with $|E(v)|>E_{0}$, then $S_{k-1}\left(E(v), v, \widetilde{\Lambda}_{k}\right) \neq \phi$, and hence $S_{k-1}\left(E(v), v, A_{k}\right)=$ $\phi$, by (3.11), for all $k>k$, with $\bar{k}=\max \left(\hat{k}, k^{\prime}\right)$. Hence the proof of Theorem 3.1 is complete.

Our proof of localization is now complete, up to the proof of Lemma 3.3 which forms the contents of the next section.

\section{Proof of the Basic Probabilistic Estimate (Lemma 3.3)}

We reduce the proof of Lemma 3.3 to two fairly simple, auxiliary lemmas, Lemma 4.3 and 4.4, which are stated below, after a brief review of some combinatorial techniques and estimates from $[15,3]$.

Let $D$ be some finite subset of $\Lambda \subseteq \mathbb{Z}^{v}$, and let $I$ be some interval of energies with the property that $I \cap\left[-E_{0}, E_{0}\right]=\phi$. Our goal is to estimate the probability, $P_{D, A}$, 
of the event that

$$
D=C_{k}^{\alpha} \subset S_{k}(E, v, \Lambda)
$$

for some integers $k$ and $\alpha$, choosing $\delta+E_{0}$ sufficiently large. For this purpose we need a bound on the "combinatorial entropy" of the set $D$. This notion is defined as follows: Let $\mathfrak{C}_{n}(D)$ be a minimal family of cubes in $\mathfrak{C}_{n}$ (defined before (2.9)) which cover $D$. Let $n(D)$ be the smallest integer such that $D$ is covered by a single cube $c \in \mathfrak{C}_{n(D)}$. (Clearly $2^{n(D)} \leqq 2$ diam $D$.) We define $V_{n}(D)$ to be the cardinality, $\left|\mathfrak{C}_{n}(D)\right|$, of $\mathfrak{C}_{n}(D)$ and set

$$
V(D)=\sum_{n=0}^{n(D)} V_{n}(D)
$$

We call $V(D)$ the combinatorial entropy of $D$.

Theorem 4.1 [3]. Let $N(V)$ be the total number of subsets $D \subset \mathbb{Z}^{v}$ such that $V(D)=V$ and $D \ni 0$. Then

$$
N(V) \leqq \exp \left[K_{v} V\right],
$$

where $K_{v}$ is some finite constant which depends only on the dimension $v$.

Example. If $D$ consists of two points separated by a distance $l$, then $V_{n}(D)=2$ for all $n$ such that $2^{n}<l$. Hence

$$
V(D)=2 \log _{2} l+\text { const. }
$$

More generally, since $V_{n}(D) \geqq 2$, for all $n<n(D)$, and $n(D) \leqq \log _{2} \operatorname{diam} D+$ const, we have

$$
V(D) \geqq 2 \log _{2} \operatorname{diam}(D)+\text { const. }
$$

We shall also need the notion of "isolated" cubes of $\mathfrak{C}_{n}(D)$. Let $b$ be given, with $1 \leqq b<2$. We define

$$
\mathfrak{C}_{n}^{\prime}(D)=\left\{c \in \mathfrak{C}_{n}(D): \operatorname{dist}\left(c, c^{\prime}\right) \geqq 2^{b n+1}, \text { for all } c^{\prime} \in \mathfrak{C}_{n}(D), \text { with } c^{\prime} \neq c\right\},
$$

and

$$
V_{n}^{\prime}(D)=\left|\widetilde{C}_{n}^{\prime}(D)\right|, \quad V^{\prime}(D)=\sum_{n=2}^{n(D)} V_{n}^{\prime}(D)
$$

The following result has been shown in [15]; see also Sect. 4 of [3].

Theorem 4.2. There is a constant $K_{v}^{\prime}$, only depending on the dimension $v$, such that

$$
V(D) \leqq K_{v}^{\prime}\left[|D|+V^{\prime}(D)\right]
$$

for all $D$.

Throughout the remainder of Sect. 4 we choose $\delta+E_{0}$ large enough and fix an arbitrary interval, $I$, of energies such that $I \cap\left[-E_{0}, E_{0}\right]=\phi$ and $|I|$ is small enough. Let $P_{D, A}$ be the probability of the event that

$$
D=C_{k}^{\alpha} \subset S_{k}(E, v, \Lambda),
$$

for some $k$ and $\alpha$ and some energy $E \in I$. Let $\chi_{D}$ be the characteristic function of this 
event. We set

$$
q_{D}=\#\left\{n: V_{n}^{\prime}(D)=1\right\} .
$$

Recall that $d_{k}=\exp \left[\beta(5 / 4)^{k}\right], k=0,1,2, \ldots ;(\operatorname{see}(1.16))$. Our principal results in this section are the following two lemmas.

Lemma 4.3. For any fixed $\beta$ and $b=5 / 3$ in (4.5)

$$
P_{D, \Lambda} \leqq \exp \left[-k_{0}\left(|D|+V^{\prime}(D)-q_{D}\right)\right],
$$

provided $\delta+E_{0}$ is large enough and $|I|$ is small enough. The constant $k_{0}=k_{0}\left(\delta, E_{0}\right)$ tends to $+\infty$, as $\delta+E_{0} \rightarrow \infty$.

Lemma 4.4. If $D=C_{i}^{\alpha} \subseteq S_{i}(E, v, \Lambda)$, for some $\alpha$ and some $i \geqq k$, and if $E$ satisfies

$$
\operatorname{dist}\left(E, \sigma\left(H_{c \cap \Lambda}\right)\right) \geqq \exp \left[-d_{k-1}^{\gamma}\right]
$$

for all $c \in \mathbb{C}=\bigcup_{m=0}^{\infty} \mathfrak{C}_{m}$, then

$$
q_{D} \leqq \frac{1}{2}\left\{|D|+V^{\prime}(D)\right\}
$$

and

$$
\operatorname{diam}(D) \geqq d_{k-1},
$$

provided $\gamma \leqq \frac{1}{2}$ is sufficiently small.

We now first prove (4.12) and then show how Lemma 3.3 follows from Lemmas 4.3 and 4.4. Subsequently, we shall prove Lemmas 4.3 and 4.4 .

Proof of (4.12). If $E$ satisfies (4.10) then

$$
\operatorname{dist}\left(\sigma\left(H_{\bar{C}_{i}^{\alpha}}\right), E\right) \geqq e^{-\sqrt{d_{i-1}}} \gg e^{-\sqrt{d_{i}}},
$$

for all $i \geqq k$, because, by (2.9), $\bar{C}_{i}^{\alpha} \in \mathbb{C}$, and $\sqrt{d_{i-1}} \geqq d_{k-1}^{\gamma}$, for $i \geqq k$ and $\gamma \leqq \frac{1}{2}$. Hence by Condition $k$, (a) and (c), Sect. 2,

$$
\operatorname{diam}\left(C_{i}^{\alpha}\right) \geqq d_{i-1} \geqq d_{k-1} .
$$

For, otherwise, $C_{i}^{\alpha}$ would really belong to $S_{j} \backslash S_{j+1}$, for some $j<i$, by the maximality condition imposed on $S_{j} \backslash S_{j+1}$. (See (2.3)-(2.6).) Clearly, (4.13) proves (4.12).

From these arguments we conclude that if $E$ satisfies $(4.10)$ and $S_{k}(E, v, \Lambda) \neq \phi$, then there exists some singular component $C_{j}^{\alpha} \subseteq \Lambda, j \geqq k$, with

$$
\operatorname{diam}\left(C_{j}^{\alpha}\right) \geqq d_{k-1} .
$$

Proof of Lemma 3.3. By the definition of $\mathfrak{B}_{k}(\Lambda, I)$, see Definition 4, in particular (3.5), we thus have

$\operatorname{Prob}\left(\mathfrak{B}_{k}(\Lambda, I)\right) \leqq \operatorname{Prob}\left\{v: \exists C_{j}^{\alpha} \subseteq \Lambda\right.$, with $k \leqq j<\infty$, and $\left.\operatorname{diam}\left(C_{j}^{\alpha}\right) \geqq d_{k-1}\right\}$

$$
\leqq \sum_{\substack{D: \operatorname{diam}(D) \geq d_{k-1} \\ D \subseteq \bar{\Lambda}}} P_{D, \Lambda} .
$$


Using Lemmas 4.3 and 4.4 , we obtain the bound

$$
\operatorname{Prob}\left(\mathfrak{B}_{k}(\Lambda, I)\right) \leqq|\Lambda| \sum_{\substack{D: \operatorname{diam}(D) \geqq d_{k-1} \\ D \ni 0}} \exp \left[-\frac{k_{0}}{2}\left(|D|+V^{\prime}(D)\right)\right] .
$$

To control the sum on the right-hand-side of (4.14) we make use of Theorems 4.1 and 4.2, thus obtaining

$$
\operatorname{Prob}\left(\mathfrak{B}_{k}(\Lambda, I)\right) \leqq|\Lambda| \sum_{V \geqq V_{k}} \exp \left[\left(K_{v}-\frac{k_{0}}{K_{v}^{\prime}}\right) V\right],
$$

where $V_{k}=\min _{D}\left\{V(D): \operatorname{diam}(D) \geqq d_{k-1}\right\}$. By (4.4),

$$
V_{k} \geqq 2 \log _{2} d_{k-1} .
$$

Since $k_{0}$ can be made arbitrarily large by choosing $\delta+E_{0}$ large and $|I|$ small, we conclude from (4.15) and (4.16) that

$$
\operatorname{Prob}\left(\mathfrak{B}_{k}(\Lambda, I)\right) \leqq|\Lambda| d_{k}^{-p}
$$

for $\delta>\delta_{0}(p)$ and $|I|$ small enough, or $I \cap\left[-E_{0}, E_{0}\right]=\phi$, for some $E_{0}=E_{0}(p, \delta)$. This completes the proof of Lemma 3.3.

Before starting the proof of Lemmas 4.3 and 4.4, we show that if $D=C_{i}^{\alpha} \subset$ $S_{i}(E, v, \Lambda)$ and $c \in \widetilde{C}_{m}^{\prime}(D)$, then

$$
\operatorname{dist}\left(\sigma\left(H_{c \cap D \cap A}\right), E\right) \leqq \exp -\sqrt{d_{j(m)}},
$$

where $j(m)$ is defined in such a way that

$$
d_{j(m)} \geqq 2^{m}>d_{j(m)-1}=d_{j(m)}^{4 / 5} .
$$

Moreover, $\overline{c \cap D}$ is the cube in $\mathfrak{C}_{n(j(m))}$ containing $c \cap D$ and satisfying

$$
\operatorname{dist}(c \cap D, \partial(\overline{c \cap D}) \backslash \partial \Lambda) \geqq 4 d_{j(m)} ;
$$

see (2.8)-(2.10). A proof of (4.18), (4.19) was given in [3], but for the convenience of the reader it is repeated here. Since $c \in \mathfrak{C}_{m}^{\prime}(D)$,

$$
\operatorname{diam}(c \cap D) \leqq 2^{m} \leqq d_{j(m)} .
$$

Hence $c \cap D$ satisfies Condition $k$, (a) at scale $j(m)$; (see (2.4)). We claim that $c \cap D$ also satisfies Condition $k$, (b) (the distance condition), i.e. (2.5). This implies that (4.18) holds, because otherwise $c \cap D=c \cap C_{i}^{\alpha}$ would be regular at scale $j(m)$ contradicting the maximality of $S_{j} \backslash S_{j+1}$, for some $j \leqq j(m)$.

Next, we verify our claim. By the definition of $\mathbb{C}_{m}^{\prime}(D)$, see (4.5), and since we have chosen $b=5 / 3$,

$$
\begin{aligned}
\operatorname{diam} D & \geqq \operatorname{dist}(c, D \backslash(c \cap D)) \\
& \geqq 2 \cdot 2^{(5 / 3) m} \\
& \geqq 2 \cdot\left(2^{m}\right)^{(5 / 4)^{2}} \\
& \geqq 2 d_{j(m)}^{5 / 4} \\
& =2 d_{j(m)+1},
\end{aligned}
$$


and we have used (4.19). Hence, by Condition $k$, (a),

$$
i \geqq j(m)+1 \text {. }
$$

By Condition $k$, (b) (see (2.5)) and (4.21),

$$
\begin{aligned}
\operatorname{dist}\left(c \cap D, S_{i} \backslash D\right) & \geqq \operatorname{dist}\left(D, S_{i} \backslash D\right) \\
& \geqq 2 d_{i+1} \\
& >2 d_{j(m)+1} .
\end{aligned}
$$

Moreover, if $C_{l}^{\alpha^{\prime}}$ is a singular component different from $D$, then $c \cap D \subset S_{l} \backslash C_{l}^{\alpha^{\prime}}$, and hence, by Condition $k$, (b),

$$
\operatorname{dist}\left(c \cap D, C_{l}^{\alpha^{\prime}}\right) \geqq 2 d_{l+1},
$$

in particular

$$
\operatorname{dist}\left(c \cap D, C_{l}^{\alpha^{\prime}}\right) \geqq 2 d_{j(m)+1},
$$

for $j(m) \leqq l \leqq i$. Equations (4.20) and (4.22)-(4.24) establish our claim.

Proof of Lemma 4.3. Suppose that

$$
D=C_{k}^{\alpha} \subset S_{k}(E, v, \Lambda), \quad \text { for some } E \in I,
$$

and

$$
V_{m}^{\prime}(D) \geqq 2,
$$

for some $m$. Then (4.18) holds for two disjoint cubes, $c_{1}$ and $c_{2}$, in $\mathfrak{C}_{m}^{\prime}(D)$. Therefore

$$
\operatorname{dist}\left(\sigma\left(H_{B_{1}}\right) \cap I, \quad \sigma\left(H_{B_{2}}\right)\right) \leqq 2 e^{-\sqrt{d_{j(m)}}},
$$

where $B_{i} \equiv\left(\overline{\left.c_{i} \cap D\right)} \cap \Lambda, i=1,2\right.$. Let $\chi_{m, c_{1}, c_{2}}(v)$ be the characteristic function of the event described in (4.26). If $m=0, c$ can be identified with a lattice site, and, since $E \in I$, the event

$$
|v(c)-E| \leqq 2 v+m_{0}, \quad \text { for some } E \in I,
$$

holds; see (2.1). Let $\chi_{0, c}$ be the characteristic function of the event described in (4.27), and let $\chi_{D}$ be the characteristic function of the event described in (4.25). Then

$$
\chi_{D}(v) \leqq \prod_{c \in \mathbb{E}_{0}(D)} \chi_{0, c}(v) \prod_{m \geqq 2} \prod_{c_{1} c_{2} \in \mathbb{C}_{m}^{\prime}(D)}^{\prime} \chi_{m, c_{1}, c_{2}}(v) .
$$

The product $\prod^{\prime}$ extends over disjoint pairs of cubes in $\mathbb{C}_{m}^{\prime}(D)$. If $\mathfrak{C}_{m}^{\prime}(D)$ contains only one cube the product is empty. For fixed $m$, the events in the supports of $\chi_{m, c_{1}, c_{2}}$ are independent, as $c_{1}, c_{2}$ ranges over disjoint pairs of distinct cubes, because

$$
(\overline{c \cap D}) \cap\left(\overline{\left.c^{\prime} \cap D\right)}=\phi\right.
$$

for $c$ and $c^{\prime}$ in $\mathbb{C}_{m}^{\prime}(D)$, with $c \neq c^{\prime}$. We note that there are at least $\left(V_{m}^{\prime}(D)-1\right) / 2$ factors in the product, $\prod^{\prime}$, on the right-hand-side of (4.28). Using Corollary 2.3 we see that

$$
\overline{\chi_{m, c_{1}, c_{2}}} \equiv \int d P(v) \chi_{m, c_{1}, c_{2}}(v) \leqq \exp \left[-\left(\frac{1}{2} \sqrt{d_{j(m)}}+k_{0}^{\prime}\right)\right],
$$

where $k_{0}^{\prime}=k_{0}^{\prime}\left(\delta, E_{0}\right) \rightarrow \infty$, as $\delta+E_{0} \rightarrow \infty$. 
As in Sect. 5 of [3] we now iterate Hölder's inequality, using that $\chi(v)^{p}=\chi(v)$, for all $p>0$, and obtain

$$
\begin{aligned}
P_{D, \Lambda} & \equiv \overline{\chi_{D}} \equiv \int d P(v) \chi_{D}(v) \\
& \leqq \prod_{c \in \mathbb{C}_{0}(D)}{\overline{\chi_{0}, c}}^{1-r}\left[\int \prod_{m>0} \prod_{c_{1}, c_{2} \in \mathbb{C}_{m}^{\prime}(D)}^{\prime} \chi_{m, c_{1}, c_{2}} d P(v)\right]^{r} \\
& \leqq \ldots \\
& \leqq \prod_{c \in \mathbb{C}_{0}(D)} \bar{\chi}_{0, c}^{1-r} \prod_{m>0} \prod_{c_{1}, c_{2}}^{\prime} \bar{\chi}_{m}, c_{1}, c_{2}(1-r)
\end{aligned}
$$

Choosing $r=0.8>2^{-1 / 2}$ we obtain from (4.29) and (4.30),

$$
\begin{aligned}
\bar{\chi}_{m, c_{1}, c_{2}}{ }^{r^{m}(1-r)} & \leqq \exp \left[-r^{m}(1-r)\left(\frac{\sqrt{2^{m}}}{2}+k_{0}^{\prime}\right)\right] \\
& \leqq e^{-k_{0}}
\end{aligned}
$$

for some $k_{0}=k_{0}\left(\delta, E_{0}\right)$, which tends to $+\infty$, as $\delta+E_{0} \rightarrow \infty$. Here we have used that $d_{j(m)} \geqq 2^{m}$; see (4.19). This completes the proof of Lemma 4.3.

Remark. Since $E$ is not fixed, we do not get any small factors from cubes $c \in \mathbb{C}_{m}^{\prime}(D)$ if $V_{m}^{\prime}(D)=1$. This yields the term $q_{D}$ on the right-hand-side of (4.9).

Proof of Lemma 4.4. Let $D$ and $E$ satisfy the hypotheses of Lemma 4.4. Inequality (4.12), i.e.

$$
\operatorname{diam} D \geqq d_{k-1}
$$

has already been proven, for $\gamma \leqq 1 / 2$; see (4.13). Next, we prove (4.11), i.e.

$$
q_{D} \leqq \frac{1}{2}\left\{|D|+V^{\prime}(D)\right\} \text {. }
$$

Let $c \in \mathfrak{C}_{m}^{\prime}(D)$. By (4.18)

$$
\operatorname{dist}\left(\sigma\left(H_{\overline{c \cap D} \cap \Lambda}\right), E\right) \leqq \exp -\sqrt{d_{j(m)}} .
$$

But by hypothesis,

$$
\operatorname{dist}\left(\sigma\left(H_{\overline{\boldsymbol{c} \cap \boldsymbol{D}} \cap \Lambda}\right), E\right) \geqq \exp \left[-d_{k-1}^{\gamma}\right]
$$

see (4.10). Therefore,

$$
\mathfrak{C}_{m}^{\prime}(D)=\phi
$$

if

$$
2^{(5 / 8) m} \geqq \sqrt{d_{j(m)}} \geqq d_{k-1}^{\gamma},
$$

and we have used (4.19). Hence, using (4.4) and (4.12),

$$
\begin{aligned}
q_{D} & \leqq C \gamma \log _{2} d_{k-1} \\
& \leqq C \gamma \log _{2} \operatorname{diam}(D) \\
& \leqq C^{\prime} \gamma V(D) \\
& \leqq C^{\prime} \gamma K_{v}^{\prime}\left[|D|+V^{\prime}(D)\right] \\
& \leqq \frac{1}{2}\left[|D|+V^{\prime}(D)\right]
\end{aligned}
$$


for $\gamma \leqq 1 /\left(2 C^{\prime} K_{v}^{\prime}\right)$. Here $C$ and $C^{\prime}$ are finite constants, and we have used Theorem 4.2. This completes the proof of Lemma 4.4 .

Completeness of point spectrum outside $\left[-E_{0}, E_{0}\right]$, for large $\delta+E_{0}$, is thus established.

\section{Localization in One Dimension}

In this section we briefly sketch how to extend our method to prove localization in the one-dimensional Anderson model at arbitrary energies and for arbitrarily weak disorder, $\delta$. Although we do not prove any new results on one-dimensional models, we feel that our method of proof is quite close to physical intuition about tunnelling and that it will extend more easily to more general problems, such as localization in a strip, than some of the previous proofs. See $[16,10,11,2,17]$, and references given there, for earlier results.

To prove localization it suffices to show that, for any sufficiently short interval, $I$, of energies,

$$
I \cap \sigma(H)=\sigma_{\mathrm{pp}}(H)
$$

where $H=-\Delta+v$ is the one-dimensional tight binding Hamiltonian; see (1.2)(1.6). In Sect. 2, (2.14)-(2.17), we have outlined a strategy for proving (5.1) based on establishing decay of the Green's function over a sufficiently large block $B_{l} \equiv[0,2 l]$, for arbitrary energies in $I$, with probability close to one. More precisely, we must show that, for all $E \in I$,

$$
\operatorname{Prob}\left\{v:\left|G_{B_{l}}(E ; x, y)\right| \leqq a<\frac{1}{2}, \text { for }|x-y| \geqq \frac{l}{2}\right\} \geqq 1-\varepsilon(l, I, a),
$$

where $y=0$, or $=2 l$, and $\varepsilon(l, I, a) \rightarrow 0$, as $l \rightarrow \infty$ and $|I| \rightarrow 0$. See (2.14). In order to prove (5.2), we must look for a way to control $G_{B_{1}}(E ; x, y)$ non-perturbatively. This is provided by the Fürstenberg theorem [18] and simple density-of-states estimates; (Theorem 2.2).

We start by expressing the Green's function $G_{B_{l}}(E ; 0,2 l)$ in terms of the solution, $\psi_{E}$, of the initial value problem

$$
\left([H-E] \psi_{E}\right)(j)=0, \quad j \geqq 0,
$$

with

$$
\psi_{E}(-1)=0 \quad \text { and } \quad \psi_{E}(0)=1 \text {. }
$$

Let $\Gamma$ be defined as in (1.13), with $\Lambda=B_{l}$. Equation (5.3) can be rewritten as $\left([H+\Gamma-E] \psi_{E}\right)(j)=\left(\Gamma \psi_{E}\right)(j)$, and hence, for $j \in B_{l}$,

$$
\psi_{E}(j)=\sum_{\left\langle i, i^{\prime}\right\rangle \in \partial B_{l}} G_{B_{l}}(E ; j, i) \psi_{E}\left(i^{\prime}\right)
$$

Setting $j=0$ and noting that $\psi_{E}\left(i^{\prime}=-1\right)=0$, we obtain the well-known identity

$$
G_{B_{l}}(E ; 0,2 l)=\psi_{E}(0) / \psi_{E}(2 l+1)=1 / \psi_{E}(2 l+1) .
$$

Next we recall the Fürstenberg theorem [18]. 
Theorem 5.1. Let $E$ be an arbitrary energy and let $\psi_{E}(j), j \in \mathbb{Z}^{+}$, be the unique solution of the initial value problem formulated in (5.3), (5.4). Suppose $\{v(j)\}, j \in \mathbb{Z}^{v}$, are i.i.d. random variables with distribution $g(v(j)) d v(j)$, where $g$ is some bounded function. Then there exists a set $\Omega_{E} \subseteq \Omega$ of potentials of full measure (i.e. $\operatorname{Prob}\left(\Omega_{E}\right)=1$ ) such that for all $v \in \Omega_{E}$,

$$
\gamma(E) \equiv \lim _{j \rightarrow \infty} \frac{1}{j} \ln \left|\psi_{E}(j)\right|,
$$

exists and is a positive constant, the Liapunov exponent, which is independent of the particular configuration $v \in \Omega_{E}$.

Remark. One can also express $\gamma(E)$ with the help of the Thouless formula [19]

$$
\gamma(E)=\int \ln \left|E-E^{\prime}\right| d N\left(E^{\prime}\right),
$$

where $d N\left(E^{\prime}\right)$ is the integrated density of states.

From (5.6) and (5.7) it is quite clear that $G_{B_{l}}(E ; 0,2 l)$ is exponentially small in $l$ with high probability. This is made precise in the following corollary.

Corollary 5.2. For an arbitrary, but fixed energy $E$ and any $\varepsilon$, with $0<\varepsilon<\gamma(E)$,

$$
\lim _{l \rightarrow \infty} \operatorname{Prob}\left\{v:\left|G_{B_{l}}(E ; 0,2 l)\right| \geqq \exp [-(\gamma(E)-\varepsilon) 2 l]\right\}=0 .
$$

Proof. Clearly

$$
\begin{aligned}
\left\{v:\left|G_{B_{l}}(E ; 0,2 l)\right|\right. & \geqq \exp [-(\gamma(E)-\varepsilon) 2 l]\} \\
& \subseteq \Omega(E, \varepsilon, l) \equiv\left\{v:\left|\frac{1}{2 l} \ln \right| G_{B_{l}}(E ; 0,2, l)|+\gamma(E)| \geqq \varepsilon\right\} .
\end{aligned}
$$

By (5.6) and Theorem 5.1,

$$
\frac{1}{2 l} \ln \left|G_{B_{l}}(E ; 0,2 l)\right|=-\frac{1}{2 l} \ln \left|\psi_{E}(2 l+1)\right|
$$

tends to $-\gamma(E)$, as $l \rightarrow \infty$, almost surely. Hence $\operatorname{Prob}(\Omega(E, \varepsilon, l)) \rightarrow \infty$, for every fixed $\varepsilon>0$ and an arbitrary, fixed energy $E$. This completes the proof.

Our main technical result in this section is the following theorem.

Theorem 5.3. Choose an energy $E_{0} \in \sigma(H)$. Let $\left\{\Delta_{l}\right\}, l=1,2,3, \ldots$, be a sequence of numbers such that $l^{4} \Delta_{l} \rightarrow 0$, as $l \rightarrow \infty$. Then, for any $\lambda$, with $0<\lambda<1$,

$$
\lim _{l \rightarrow \infty} \operatorname{Prob}\left\{v: \sup _{\left|E-E_{0}\right| \leqq \Delta_{l}}\left(\left|G_{B_{l}}(E ; 0, j)\right|+\left|G_{B_{l}}(E ; j, 2 l)\right|\right)<\lambda \text {, for every } j \in\left[\frac{l}{2}, \frac{3 l}{2}\right]\right\}=1 \text {. }
$$

Proof. We choose some $\varepsilon$, with $0<\varepsilon<\gamma\left(E_{0}\right)$, and define two subsets of potentials by

$$
\begin{aligned}
\Omega^{(1)}\left(E_{0}, \varepsilon, l\right)= & \left\{v:\left|G_{[0, j]}\left(E_{0} ; 0, j\right)\right|+\left|G_{[j, 2 l]}\left(E_{0} ; j, 2 l\right)\right|\right. \\
& \left.\leqq 2 e^{-\left(\gamma\left(E_{0}\right)-\varepsilon\right) l / 2}, \text { for all } j \in\left[\frac{l}{2}, \frac{3 l}{2}\right]\right\}
\end{aligned}
$$


and

$$
\Omega^{(2)}\left(E_{0}, l\right)=\left\{v: \operatorname{dist}\left(\sigma\left(H_{B_{l}}\right), E_{0}\right) \geqq 2 l^{-2}\right\} .
$$

It follows from Corollary 5.2 (with $B_{l}$ replaced by $[0, j],[j, 2 l]$, respectively, that

$$
\operatorname{Prob}\left(\Omega^{(1)}\left(E_{0}, \varepsilon, l\right)\right) \rightarrow 1, \quad \text { as } l \rightarrow \infty,
$$

as one verifies easily.

Moreover, from Theorem 2.2 (Wegner's bound on the density of states) we infer that

$$
\operatorname{Prob}\left(\Omega^{(2)}\left(E_{0}, l\right)\right) \rightarrow 1, \quad \text { as } \quad l \rightarrow \infty
$$

Hence

$$
\operatorname{Prob}\left(\Omega^{(1)}\left(E_{0}, \varepsilon, l\right) \cap \Omega^{(2)}\left(E_{0}, l\right)\right) \rightarrow 1, \quad \text { as } \quad l \rightarrow \infty .
$$

By the second resolvent identity,

$$
\begin{aligned}
G_{B_{l}}\left(E_{0} ; 0, j\right) & =G_{[0, j]}\left(E_{0} ; 0, j\right)+\left\{G_{[0, j]}\left(E_{0}\right) \Gamma^{(j)} G_{B_{l}}\left(E_{0}\right)\right\}(0, j) \\
& =G_{[0, j]}\left(E_{0} ; 0, j\right)\left\{1+G_{B_{l}}\left(E_{0} ; j+1, j\right)\right\},
\end{aligned}
$$

where $\Gamma_{i i^{\prime}}^{(j)}=1$, if $i=j$ and $i^{\prime}=j+1=0$, otherwise. Similarly

$$
G_{B_{l}}\left(E_{0} ; j, 2 l\right)=G_{[j, 2 l]}\left(E_{0} ; j, 2 l\right)\left\{1+G_{B_{l}}\left(E_{0} ; j, j-1\right)\right\} .
$$

Suppose now that $v \in \Omega^{(1)}\left(E_{0}, \varepsilon, l\right) \cap \Omega^{(2)}\left(E_{0}, l\right)$. Then we derive from (5.13) and (5.14), using (5.10) and (5.11) that

$$
\left|G_{B_{l}}\left(E_{0} ; 0, j\right)\right| \leqq e^{-\left(\gamma\left(E_{0}\right)-\varepsilon\right) l / 2}\left(1+\frac{l^{2}}{2}\right)
$$

and

$$
\left|G_{B_{l}}\left(E_{0} ; j, 2 l\right)\right| \leqq \mathrm{e}^{-\left(\gamma\left(E_{0}\right)-\varepsilon\right) l / 2}\left(1+\frac{l^{2}}{2}\right) .
$$

Thus, using the first resolvent identity

$$
G_{B_{l}}\left(E ; i, i^{\prime}\right)=G_{B_{l}}\left(E_{0} ; i, i^{\prime}\right)+\left(E-E_{0}\right)\left(G_{B_{l}}(E) G_{B_{l}}\left(E_{0}\right)\right)\left(i, i^{\prime}\right),
$$

and (5.10), (5.11), we obtain the bound

$$
\left|G_{B_{l}}(E ; 0, j)\right|+\left|G_{B_{l}}(E ; j, 2 l)\right| \leqq 2\left(1+\frac{l^{2}}{2}\right) e^{-\left(\gamma\left(E_{0}\right)-\varepsilon\right) l / 2}+2 \Delta_{l} l^{4}<\lambda
$$

for $l$ large enough and $v \in \Omega^{(1)}\left(E_{0}, \varepsilon, l\right) \cap \Omega^{(2)}\left(E_{0}, l\right)$. Since $\varepsilon<\gamma\left(E_{0}\right)$ and $\Delta_{l} l^{4} \rightarrow 0$, as $l \rightarrow \infty$, the proof of Theorem 5.3 is complete.

\section{Remarks.}

(1) Suitable choices for $\Delta_{l}$ are $\Delta_{l}=\exp \left[-\operatorname{const} \gamma\left(E_{0}\right) l\right]$, or $\Delta_{l}=$ const $l^{-n}$, with $n>4$.

(2) Clearly, (5.12) and (5.15) prove our basic estimate (2.14). Hence localization can be obtained by the techniques outlined in Sect. 2, (2.14)-(2.17). The details are very similar to the arguments in Sects. 3 and 4 . We note that, since $\gamma\left(E_{0}\right)>0$, for 
all $E_{0} \in \sigma(H)$, and arbitrarily small disorder, it follows that

$$
\sigma(H)=\sigma_{\mathrm{pp}}(H), \quad \sigma_{\mathrm{ac}}(H)=\sigma_{\mathrm{sc}}(H)=\phi,
$$

which is the expected result. See also $[16,10,11,3,12,17]$, and for a review see [8].

\section{Some Comments, Extensions and Open Problems}

In this section we sketch some further results and various further applications of our techniques.

6.1. Connection Between $\sigma\left(H_{\Lambda}\right)$ and $\left\{E: S_{k}(E, v, \Lambda) \neq \phi\right\}$. It is possible to modify the inductive definition of $S_{k}=S_{k}(E, v, \Lambda)$ as follows. We set

$$
S_{k+1}=S_{k} \backslash \bigcup_{\alpha} C_{k}^{\alpha},
$$

where $\bigcup_{\alpha} C_{k}^{\alpha}$ is a maximal union of components, $C_{k}^{\alpha}$, which are regular at the $k^{\text {th }}$ scale, in the sense of the following

Modified Condition $k$ :

(a) $C_{k}^{\alpha} \subseteq \Lambda$, and diam $C_{k}^{\alpha} \leqq d_{k}$,

(b) $\operatorname{dist}\left(C_{k}^{\alpha}, S_{k} \backslash C_{k}^{\alpha}\right) \geqq 2 d_{k+1}$,

(c) There exists a subset $\widehat{C}_{k}^{\alpha}$, satisfying

$$
\widehat{C}_{k}^{\alpha} \subseteq \Lambda, \quad 4 d_{k} \leqq \operatorname{dist}\left(C_{k}^{\alpha}, \partial \widehat{C}_{k}^{\alpha} \backslash \partial \Lambda\right) \leqq 8 d_{k},
$$

such that

$$
\operatorname{dist}\left(\sigma\left(H_{\hat{C}_{k}^{\alpha}}\right), E\right) \geqq e^{-\sqrt{d_{k}}}
$$

Note that the subset $\hat{C}_{k}^{\alpha}$ of $\Lambda$ for which (6.2) holds may be $(k-1)$-admissible. More precisely, if $D$ is a subset of $\Lambda$ satisfying (a) and (b) of our modified condition $k$, but $D \neq C_{l}^{\alpha}$, for all $l=1, \ldots, k$, and all $\alpha$, then there exists a $(k-1)$-admissible set $\hat{D}$ satisfying (6.1) such that

$$
\operatorname{dist}\left(\sigma\left(H_{\hat{D}}\right), E\right)<e^{-\sqrt{d_{k}}}
$$

Moreover,

$$
\operatorname{dist}\left(\sigma\left(H_{\bar{D}}\right), E\right)<e^{-\sqrt{d_{k}}}
$$

where

$$
\bar{D}=\left\{j: \operatorname{dist}(j, D) \leqq 4 d_{k}\right\} \cap \Lambda .
$$

All arguments and results of [3] go through for this modified definition of $\left\{S_{k}\right\}$, $k=0,1,2, \ldots$. The advantage of the modified definition of $S_{k}$ is that it typically yields smaller sets, $S_{k}$, of singular sites than the definition used in this paper and in [3] and that the set of "resonant energies"

$$
\mathfrak{R}_{k}(\Lambda, v) \equiv\left\{E: S_{k-1}(E, v, \Lambda) \neq \phi\right\}
$$

is statistically highly correlated with $\sigma\left(H_{\Lambda}\right)$, as one might expect. More precisely, the 
following result holds. Let

$$
\begin{aligned}
& \mathfrak{B}_{k}^{\prime}(\Lambda, I) \equiv\left\{v: S_{k-1}(E, v, \Lambda) \neq \phi, \quad\right. \text { and } \\
&\left.\cdot \operatorname{dist}\left(\sigma\left(H_{\Lambda}\right), E\right) \geqq \exp \left[-d_{k-1}^{\gamma}\right], \text { for some } E \in I\right\} .
\end{aligned}
$$

Proposition. Let $E_{0}+\delta$ be large enough. Then for sufficiently small $\gamma$ and any compact interval $I$ with $I \cap\left[-E_{0}, E_{0}\right]=\phi$ and $|I|$ small enough,

$$
\operatorname{Prob}\left(\mathfrak{B}_{k}^{\prime}(\Lambda, I)\right) \leqq|\Lambda| d_{k}^{-p},
$$

where $p \rightarrow \infty$, as $E_{0}+\delta \rightarrow \infty$.

This proposition could be used as an alternative to Lemma 3.3 in our analysis of localization. However, while this proposition makes a simpler and more intuitive statement than Lemma 3.3, its proof is considerably harder. (Since it may be of some interest in its own right, we state it here without proof.)

6.2. Other Physical Systems Exhibiting Localization. In [3,5] and in this paper we have analyzed a system with diagonal disorder, namely a quantum mechanical particle in a random potential. There are, however, plenty of disordered, linear dynamical systems with off-diagonal disorder. It is therefore interesting to note that our techniques extend to such systems in a straightforward manner, under suitable assumptions on the distribution of the off-diagonal disorder.

A typical example is an infinite array of coupled harmonic oscillators: a classical oscillator with coordinates $\vec{x}_{j} \in \mathbb{R}^{n}$ is attached at every site $j \in \mathbb{Z}^{v}$. The equations of motion of these oscillators are given by

$$
\ddot{\vec{x}}_{j}=-\stackrel{\circ}{j}_{j}^{2} \vec{x}_{j}+\sum_{j^{\prime}} f_{j^{\prime} j}\left(\vec{x}_{j^{\prime}}-\vec{x}_{j}\right)
$$

where $f_{j^{\prime} j}=0$, unless $\left|j^{\prime}-j\right|=1$, for example. The variables $\stackrel{\circ}{j}_{j}$ and $f_{j^{\prime} j}$ may be random variables with distributions $d \lambda\left(\stackrel{\circ}{j}_{j}\right)$ and $d \rho\left(f_{j^{\prime} j}\right)$, where

$$
d \rho\left(f_{j^{\prime} j}\right)=h\left(f_{j^{\prime} j}\right) d f_{j^{\prime} j},
$$

with $h$ a bounded function supported in $[0, \infty)$. For example

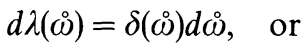

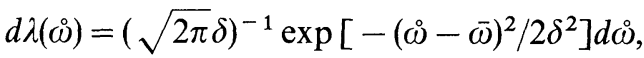

and

$$
\begin{aligned}
& h(f)=\frac{1}{N} \chi_{[0, N]}(f), \text { or } \\
& h(f)=\left(\sqrt{\frac{\pi}{2}} N\right)^{-1} \exp \left[-f^{2} / 2 N^{2}\right] \theta(f) .
\end{aligned}
$$

The model specified in (6.3), (6.4) describes harmonic vibrations of a crystal lattice. In order to solve the equations of motion (6.3), we must analyze the Jacobi matrix $\Omega^{2}$ defined by

$$
\Omega_{i j}^{2}=\left\{\begin{array}{l}
f_{i j}, \quad \text { if }|i-j|=1 \\
-\left(\stackrel{\circ}{i}^{2}+\sum_{j:|j-i|=1} f_{i j}\right), \quad \text { if } \quad i=j \\
0, \text { otherwise. }
\end{array}\right.
$$


This is a "tight binding Hamiltonian" with diagonal disorder given by $\left\{-\left(\omega_{i}^{2}+\sum_{j:|j-i|=1} f_{i j}\right)\right\}, i \in \mathbb{Z}^{v}$, and off-diagonal disorder given by $\left\{f_{i j}\right\}$. The spectrum of $\Omega$ corresponds to the normal frequencies and the generalized eigenfunctions of $\Omega$ are the normal modes of (6.3). (We define $\Omega$ to be the positive root of $\Omega^{2}$.) If $d \lambda$ is given by (6.4), i.e. $\omega_{j} \equiv 0$, one may prove that, for $\omega_{0}+N$ large enough,

$$
\sigma_{\mathrm{ac}}(\Omega) \cap\left(\omega_{0}, \infty\right)=\sigma_{\mathrm{sc}}(\Omega) \cap\left(\omega_{0}, \infty\right)=\phi .
$$

However, there is always an extended generalized eigenstate of $\Omega, \vec{x}_{j}^{(\omega=0)}=$ const, corresponding to a generalized eigenvalue $\omega=0$, and one expects that the localization length diverges to $+\infty$, as $\omega \searrow \omega_{0}$, with $\omega_{0}=0$ in one dimension. (For $d \lambda(\omega)$ as in (6.5) the entire spectrum of $\Omega$ may be pure point if $\delta$ is large enough.) If $\omega_{0}+N$ is sufficiently large and $\omega$ is a discrete eigenvalue of $\Omega$, with $\omega>\omega_{0}$, then the corresponding normal modes $\left\{\vec{x}_{j}^{(\omega)}, \vec{y}_{j}^{(\omega)}\right\},\left(\vec{y}_{j}=\dot{\vec{x}}_{j}\right), j \in \mathbb{Z}^{v}$, decay exponentially in $|j|$. It is convenient to complexify the phase space by setting $\vec{\xi}_{j}=\vec{x}_{j}+\overrightarrow{i y}_{j}$. Let $\left\{\vec{\xi}_{j}^{0}\right\}$ be an initial condition localized near $j=0$, with the property that

$$
E_{\Omega}\left(\left[0, \omega_{0}\right]\right) \vec{\xi}^{0}=0,
$$

where $E_{\Omega}(\cdot)$ is the spectral resolution of $\Omega$. Let $\vec{\xi}_{j}(t)=\left(e^{i \Omega t} \vec{\xi}^{0}\right)_{j}$ be a solution of $(6.3)$ with initial conditions $\vec{\xi}^{0}$. Then

$$
\sum_{j \in \mathbb{Z}^{v}}|j|^{2}\left|\vec{\xi}_{j}(t)\right|^{2} \leqq \text { const }
$$

uniformly in $t$. In the one-dimensional case these results have been established in [12]. For $v>1$, one could, in principle, use the techniques of this paper and of $[3,5,9]$ to give proofs.

Another interesting application of localization theory concerns the propagation of electromagnetic waves in a medium with randomly distributed dielectric constant. (For simplicity we suppose that the magnetic permeability $\mu=1$.) This problem is basically just the continuum limit of the problem formulated in (6.3). However, in this case, the continuum problem is not a simple extension of the lattice problem, as one may easily understand. We believe that the mathematical methods of this paper and of $[3,5,9]$ are relevant for the analysis of Maxwell's equations if the dielectric constant $\varepsilon$ is of the form

$$
\varepsilon(\vec{x})=\varepsilon_{p}(\vec{x})+\varepsilon_{s}(\vec{x})>0, \quad \vec{x} \in \mathbb{R}^{3},
$$

where $\varepsilon_{p}$ is a smooth periodic function, and $\varepsilon_{s}$ is a smooth stochastic perturbation with the properties that $\varepsilon_{s}(\vec{x})$ and $\varepsilon_{s}\left(\vec{x}^{\prime}\right)$ are statistically independent for $\left|\vec{x}-\vec{x}^{\prime}\right|>$ $r>0$ and that $\overline{\varepsilon_{s}^{n}(\vec{x})}$ is small. However, we have not looked into any details. There are certain limiting cases of the problem described here which can be analyzed quite easily. We consider an optical medium with a frequency-independent random index of refraction, $n(\vec{x})$. We suppose that $\sup |\operatorname{grad} n(\vec{x})|$ is small. We study the propagation of light of fairly high frequency. In the absence of external currents the 
electric field then satisfies approximately the equation

$$
\left(\frac{n(\vec{x})^{2}}{c^{2}} \frac{\partial^{2}}{\partial t^{2}}-\Delta\right) \vec{E}(\vec{x}, t)=0
$$

or, after Fourier transformation in $t$,

$$
\left(-\left(\frac{\omega}{c}\right)^{2} n(\vec{x})^{2}-\Delta\right) \hat{\vec{E}}(\vec{x}, \omega)=0, \omega \in \mathbb{R} .
$$

The analysis of this equation is still quite difficult, in general. There are, however, special cases which appear to be accessible.

(a) $n(\vec{x})=n_{p}(\vec{x})+n_{s}(\vec{x})>0$,

where $n_{p}$ is a smooth periodic function, and $n_{s}$ is a small stochastic perturbation of large disorder; (same properties as $\varepsilon_{s}$ ). If $n_{s}=0$, then there are intervals of forbidden frequencies, $\omega$, for which (6.7) has no solution. If $n_{s}$ is now turned on one expects that there are discrete sets of frequencies inside a forbidden band of the periodic problem for which (6.7) has localized solutions (standing waves).

(b) Let $n(\vec{x}) \equiv n(x, y)$ be independent of $z$. Let $\phi$ be a component of $\hat{\vec{E}}$ and define $\Delta_{\perp}$ to be given by $\left(\partial^{2} / \partial x^{2}\right)+\left(\partial^{2} / \partial y^{2}\right)$. Then Eq. (6.7) becomes, after Fourier transformation in $z$,

$$
\left[\left(-\Delta_{\perp}-\left(\frac{\omega}{c}\right)^{2} n(x, y)^{2}\right) \phi\right](x, y, ; k, \omega)=-k^{2} \phi(x, y, ; k, \omega) .
$$

This is a problem to which our methods can, in principle, be applied directly. We predict that if $n(x, y)$ is random, with correlations of finite range and "smooth" distribution, and $\omega$ is sufficiently large, the spectrum of (wave vectors, $k$, of)

$$
-\Delta_{\perp}-\left(\frac{\omega}{c}\right)^{2} n(x, y)^{2}
$$

is pure point, and the eigenmodes of (6.8) are exponentially well localized in the $(x, y)$ plane. Hence electromagnetic waves of high frequency propagating in the $z$-direction do not spread in the $x$ and $y$ directions.

Interesting problems in one-dimensional localization theory concern, for example, random Schrödinger operators with a potential whose distribution is not absolutely continuous, or the theory of wave guides with stochastic boundaries.

Acknowledgements. Two of us, F.M. and E.S., gratefully acknowledge the collaboration and discussions with G. Jona-Lasinio which were instrumental in clarifying the role of hierarchical potential configurations in the localization problem.

We thank R. Stora and K. Osterwalder for their hospitality at the 1984 Les Houches summer school "Critical Phenomena..." where the basic outline of our results was worked out.

J. F. and T. S. thank the Institute for Advanced Study, where this paper was completed, for kind hospitality. 


\section{References}

1. Anderson, P.: Absence of diffusion in certain random lattices, Phys. Rev. 109, 1492 (1958)

2. Kunz, H., Souillard, B.: Sur le spectre des opérateurs aux différences finies aléatoires. Commun. Math. Phys. 78, 201 (1980)

3. Fröhlich, J., Spencer, T.: Absence of diffusion in the Anderson tight binding model for large disorder or low energy. Commun. Math. Phys. 88, 151 (1983)

4. Fröhlich, J., Spencer, T.: A rigorous approach to Anderson localization. Phys. Reports 103, 9 (1984); Fröhlich, J., Spencer, T.: Mathematical theory of Anderson localization, proceedings of the I.A.M.P. Conference at Boulder, August 1983. Physica 124A, 303 (1984)

5. Martinelli, F., Scoppola, E.: Remark on the absence of absolutely continuous spectrum in the Anderson model for large disorder or low energy. Commun. Math. Phys., 97, 465 (1985)

6. Berezanskii, J. M.: Expansion in eigenfunctions of selfadjoint operators. Transl. Math. Monogr. 17, A.M.S. 1968; Simon, B.: Schrödinger semigroups, Bull. Am. Math. Soc., New Ser. 7, 447 (1983)

7. Jona-Lasinio, G., Martinelli, F., Scoppola, E.: Multiple tunnelings in $d$-dimension: A quantum particle in a hierarchical potential, Ann. Inst. Henri Poincaré 42, No. 1, 73-108 (1985); A quantum particle in a hierarchical potential with tunneling over arbitrarily large scales, J. Phys. A17, (1984)

8. Spencer, T.: The Schrödinger equation with a random potential - a mathematical review, to appear in the proceedings of the 1984 Les Houches summer school "Critical Phenomena...", Osterwalder, K., Stora, R. eds.

9. Wegner, F.: Bounds on the density of states in disordered systems. Z. Phys. B22, 9 (1981)

10. Wegner, F.: Z. Phys. B14, 273 (1975)

11. Gol'dsheid, Ya., Molchanov, S., Pastur, L.: Pure point spectrum of stochastic one-dimensional Schrödinger operators. Funct. Anal. Appl. 11, 1 (1977).

12. Delyon, F., Kunz, H., Souillard, B.: One-dimensional wave equation in disordered media. J. Phys. A16, No. 1, 25 (1983)

13. Holden, H., Martinelli, F.: Absence of diffusion near the bottom of the spectrum for a Schrödinger operator on $L^{2}\left(\mathbb{R}^{v}\right)$. Commun. Math. Phys. 93, 197 (1984)

14. Edwards, S., Thouless, D.: Regularity of the density of states in Anderson's localized electron model, J. Phys. C4, 453 (1971); Constantinescu, F., Fröhlich, J., Spencer T.: Analyticity of the density of states and replica method for random Schrödinger operators on a lattice. J. Stat. Phys. 34, 571 (1984)

15. Fröhlich, J., Spencer, T.: The Kosterlitz-Thouless transition in two-dimensional abelian spin systems and the Coulomb gas. Commun. Math. Phys. 81, 527 (1981)

16. Ishii, K.: Localization of eigenstates and transport phenomena in the one-dimensional disordered systems. Suppl. Progr. Theor. Phys. 53, 77 (1973)

17. Carmona, R.: Exponential localization in one-dimensional disordered systems. Duke Math. J. 49, 191 (1982)

18. Fürstenberg, H.: Trans. Am. Math. Soc. 108, 377 (1963)

19. Thouless, D. J.: J. Phys. C5, 77 (1972).

Communicated by A. Jaffe

Received March 4, 1985 